\title{
Assistive Technology Outcomes Research: Contributions to Evidence-Based Assistive Technology Practice
}

All over the world a vast number of persons with disability use assistive devices in order to enable everyday activities and to improve their quality of life (QoL). The development of assistive technologies (AT) rapidly increases the range of solutions for users. There is, however, a scarcity of knowledge concerning the effectiveness of different AT, e.g. which types of devices and services function best for which groups of users and during what circumstances? Such knowledge is of great importance and in demand by persons with disability in need of an assistive device; practitioners delivering AT services, e.g. occupational therapists, speech pathologists, and rehabilitation engineers; and service providers such as public administrations, private assistive technology services, and politicians. Similar challenges are seen within health care and other public services. This is why in the 1990s the notion of evidence-based medicine was launched by a research group led by David Sackett at McMaster University in Canada. The aim was to increase the efficiency and effectiveness of treatments and since then evidencebased practice (EBP) has gained tremendous attention within health care and at a later time also within social services. Over the years, EBP has been subject of criticism in turn leading to further development and is now seen as a contribution to clinical reasoning alongside the patient's preferences and the clinician's experiences when making decisions about health care interventions. In addition, in some countries scientific evidence of interventions' effectiveness is used for priority setting purposes. Thus, it is urgent that the scientific evidence is trustworthy and up-to-date. This poses several research challenges in general, and for AT outcomes research a number of specific challenges, some of which are highlighted in this Special Issue of Technology and Disability.

When assessing the quality of evidence a hierarchy of research designs is utilised. Most hierarchies state that conclusions based on systematic reviews of randomized controlled trials (RCTs) are considered to provide the highest level of evidence about the effectiveness of an intervention. Systematic reviews use objective means of searching the literature and apply predetermined inclusion and exclusion criteria, critical appraisal, data extraction and synthesis to formulate findings. The RCT design is considered to be the most trustworthy design, as this design minimises the risk of bias and increases the internal validity. However, use of the design has a number of limitations, e.g. RCTs cannot always be used due to ethical reasons, because external validity is often poor since results do not always apply to real life situations, and because RCTs are too expensive. The first article by Anttila and colleagues in this Special Issue of Technology and Disability reports on a comprehensive overview of systematic reviews of effectiveness of AT and additionally assesses the research quality. In the light of the difficulties in applying RCT, the authors expected to identify only a few systematic reviews, but ended up with 44 articles, giving an overview of the state of the art. On the one hand more articles than expected were published, but on the other hand no articles about the effectiveness of most AT were found. Especially evidence on AT for children and young people was missing, and likewise outcomes regarding daily activities and participation at work, home and during leisure time were rarely investigated. Most available reviews concerned information and communication technology. The quality of the ev- 
idence was in many instances found to be low when using the established system for assessing research quality 'Grading of Recommendations Assessment, Development and Evaluation' (GRADE); in some cases because of the research being poorly performed, but in other cases because it was not possible to carry out RCTs, and due to the hierarchy of research designs the quality of the research was then placed on a low quality level. Based on this, the authors point out the need to develop new methods for assessing study quality.

The traditional hierarchy of research designs mainly base the assessment of the quality of evidence on internal validity, while in EBP external validity is equally important. In the second article, Vincent and Routhier address aspects of AT outcome studies' external validity. Based on the Disability Creation Process model and by means of examples of AT outcome studies, the authors investigate what kinds of research designs are used in research concerning activity outcomes in real life situations, i.e. life habits. Vincent and Routhier report that most study designs are inadequate to practitioners and subsequently the authors put proposals for future research design in AT outcomes research forward. Especially for situations when control groups or large samples are not possible - situations that often occur within AT outcomes research.

In rehabilitation research it has often been criticised that, in reality, it is not known what the active ingredients of the interventions in outcome studies consist of, the so-called 'black box' phenomenon. Likewise, in AT service delivery most interventions do not only comprise an assistive device, but mostly (perhaps always) also AT services, such as analysis of needs, selection of device, inclusion of users in the service delivery process, etc. When the aspects of an AT intervention are unknown, a service delivery practitioner, the user or anyone interested in AT intervention results, cannot know what the outcomes are results of, how to explain the outcomes, and whether the results of outcome studies are applicable to AT praxis. The third article by Lenker and colleagues addresses this issue by suggesting that classification of AT services provides a basic framework for describing AT interventions clearly. Based on assessment of four existing classifications of AT services' ability to support outcomes research, the authors conclude that none of the classifications adequately operationalize the service delivery steps in terms of intensity, duration, frequency, or other indicators of service quality as required for outcomes research in order to avoid the "black box" phenomenon. Furthermore, the authors suggest a preliminary clas- sification of services, which can be considered a major contribution to future development of a common international classification of AT services to be used in outcomes research to adequately document an AT intervention.

In the fourth article, Hoenig and colleagues are also concerned with evidence-based AT service delivery, but from a different angle. In the AT service delivery process it may be of great importance for the selection of AT interventions to be able to predict whether a device will be for short- or long-term use as this has implications for several steps in the service delivery process in terms of e.g. selection of type of device and amount of training. If such knowledge is at hand, the practitioner will be able to tailor the intervention most effectively when delivering AT services and use the resources for those who have the greatest need. Hoenig et al. very convincingly present a study, which shows that it is possible to provide evidence-based prediction for short-term use of a new wheeled mobility device based on information likely to be readily available at the beginning of the service delivery process. Even though the study cannot be directly used in practice as more studies are required in order to create and validate a predictive model, the results are very encouraging and promising, and the study shows the importance of outcomes research in order to reach the goal of EBP.

By far, most outcomes research methodology so far has dealt with instruments for outcomes measurement. In spite of that, there are still many unresolved issues, e.g. the studies by Anttila et al. and Vincent and Routhier show that a major drawback in the possibility of comparisons of outcomes studies is that standardised instruments are not always used, which in part may be because none are available. Compared to a few years ago, however, instrument development has progressed to the point where more than one instrument is available. Instead we now need to know which instrument to use in a given situation. The fifth article brings us forward by comparing two instruments specifically designed to measure AT outcomes in special education. Watson and Smith find, by administering the two instruments in parallel that one of the instruments is more sensitive to change caused by AT interventions than the other, and in addition that it is less time-consuming to administer, while the other instrument's psychometric properties are more extensively investigated. The article thus contributes considerably in assisting practitioners in selecting the most appropriate outcomes measurement instrument in connection with EBP.

In the last article two instruments are compared in a similar fashion. The instruments are used for obtaining 
QALY-weights for QALY calculations (QALY stands for Quality Adjusted Life Years) and are generic instruments, i.e. not disease or impairment specific, that concern the measurement of QoL. The QALY is a generic outcome that can be used for comparing different interventions with each other and is of great importance in health economic studies that aim to study the costeffectiveness of interventions. Persson and Husberg set out, within an AT setting, to compare two common QoL instruments that can be used to obtain the QALY weight. The authors report on differing results on costeffectiveness depending on choice of instrument and type of AT intervention. This study shows that the choice of instrument for QALY calculations is highly important as it could lead to different cost-effectiveness ratios, and these different results could affect decisions on funding and resource allocation.

We hope that this Special issue will inspire the continuation of excellent AT intervention outcomes research. We wish to thank all the authors of this Special Issue of Technology and Disability for their very valuable contributions, which we hope will help advance the field of AT outcomes research and EBP. We also wish to thank Dr. Gert Jan Gelderblom, our colleague from the AAATE Special Interest Group on AT Outcome Assessment, for valuable support and discussions during the initial phases of producing the Special issue.

Guest Editors

Åse Brandt

Danish Centre for Assistive Technology Department of Research and Development

Århus, Denmark

E-mail: aab@hmi.dk

Jenny Alwin

Center for Medical Technology Assessment

Division of Health Care Analysis

Department of Medical and Health Sciences

Linköping University

Linköping, Sweden

E-mail: jenny.alwin@liu.se 